\title{
Variations in the rancid-flavor compounds of human breastmilk under general frozen- storage conditions
}

\author{
Hsiao-Ying Hung ${ }^{1}$, Yu-Yun Hsu' ${ }^{1}$ Pei-Fang Su² and Ying-Ju Chang ${ }^{1,3^{*}}$
}

\begin{abstract}
Background: Human breastmilk provides the best nutrition for infants. When women or infants have difficulties in breastfeeding directly, breastmilk is usually pumped and frozen for later use. However, while frozen, breastmilk may develop a rancid flavor, which induces infant feeding stress and raises the mothers' concerns about the quality of frozen breastmilk. Nevertheless, few studies have investigated the variations in the compounds that cause the rancid flavor of breastmilk during frozen storage.

Methods: A repeated-measures design was adopted to quantify the variations in rancid-flavor compounds, namely acid value (AV), total free fatty acids (FFAs), and short-and intermediate-chain FFAs of breastmilk during frozen storage. Breastmilk was obtained from ten healthy mothers of full-term infants and each milk sample was divided into three aliquots: fresh, 7-day frozen and 30-day frozen samples. The fresh samples were immediately analyzed, while the others were frozen in a domestic fridge within a temperature range of -15 to $-18{ }^{\circ} \mathrm{C}$ and analyzed 7 and 30 days later.
\end{abstract}

Results: The rancid-flavor compounds of the breastmilk, namely AV, total FFAs and intermediate-chain FFAs, significantly increased with storage time, all of which reached the sensory threshold for detecting the rancid flavor of milk. In addition, the FFAs of the breastmilk samples frozen for 7 days far exceeded the detection threshold for unpleased rancid flavor, while the 30-day samples were higher than the intolerable level for most people.

Conclusions: This study revealed that the human breastmilk develops a rancid flavor during frozen storage. Therefore, we recommend that when infants refuse thawed milk, mothers can try to provide freshly expressed milk whenever possible or provide breastmilk frozen for less than 7 days. Future studies could explore the methods for slowing breastmilk lipolysis to maintain its fresh flavor.

Keywords: Human milk, Frozen breastmilk, Rancid-flavor, Frozen storage, Milk lipolysis

\section{Background}

Fresh human breastmilk is rich in nutrients and immune antibodies, making it the ideal food for infants [1]. However, sometimes breastmilk must be pumped and stored for later use, such as when infants are prematurely born or sick in the hospital; when mothers must return to work or separated away their babies for other reasons;

\footnotetext{
* Correspondence: yxc2@mail.ncku.edu.tw

${ }^{1}$ Department of Nursing \& Institute of Allied Health Sciences, College of Medicine, National Cheng Kung University, No. 1, Daxue Rd., East Dist., Tainan, Taiwan

${ }^{3}$ Nursing Department, National Cheng Kung University Hospital, Tainan, Taiwan

Full list of author information is available at the end of the article
}

or even when mothers simply want to maintain or increase their milk supply.

The recommendations for storing the expressed breastmilk vary depending on the storage conditions. The general storage guidelines suggests that breastmilk can be kept at room temperature lower than $26^{\circ} \mathrm{C}$ for 4 6 hours, stored in a refrigerator at temperatures colder than $4^{\circ} \mathrm{C}$ for 3-8 days, and in a freezer at temperatures $-17 \sim-20{ }^{\circ} \mathrm{C}$ for at least 3 months $[2,3]$. Accordingly, when breastmilk is expected to be consumed later than 3 to 8 days, freezing is a common practice for mothers handling their milk at home. 
Breastmilk freezing is believed to be safe since the major nutrients are preserved and the growth of harmful bacteria is retarded $[4,5]$. However, a study reported that feeding premature infants (postmenstrual age nearly 37 weeks) with milk frozen for approximately one month induces more stress responses, e.g., vomiting, coughing, and gagging, when compared with fresh milk [6]. Despite the lack of evidence indicating that frozen milk harms infants' health, such feeding stresses inhibit infants from obtaining adequate breastmilk nutrition and raise mothers' concerns about the quality and safety of frozen breastmilk.

The premature infants' feeding-stress responses observed in that study were reported to be related to the rancid flavor of the frozen milk $[7,8]$, which was caused by the products of milk lipolysis [2]. Milk lipolysis refers to the milk lipids being hydrolyzed by lipase into free fatty acids (FFAs) and glycerol. Large amounts of FFAs accumulation, especially volatile short and intermediatechain fatty acids (C4:0-C12:0), cause a rancid flavor in milk [9]. Generally, milk lipids exist in the form of fat globules of 0.1 to $20 \mu \mathrm{m}$ in diameter, which are enclosed by a fat globule membrane. This membrane separates the fat globules from the lipase in the milk serum, thereby avoiding the occurrence of lipolysis. However, the freezing process results in the crystallization of milk lipids and damages the fat globule membrane, allowing milk lipolysis to occur [7, 9]. Previous studies have indicated that only when milk is stored at temperatures below $-70{ }^{\circ} \mathrm{C}$ or pasteurized before freezing will the milk lipase activity be inhibited, which delays the process of milk lipolysis $[10,11]$. Be that as it may, the temperature range of typical domestic freezer is generally $-18^{\circ} \mathrm{C}$ to $-20^{\circ} \mathrm{C}$ and human breastmilk frozen storage at home is not usually pasteurized before freezing, and so the rancid-flavor development of breastmilk due to lipolysis is generally inevitable under the typical frozen-storage regime.

Although previous studies have reported the occurrence of milk lipolysis during frozen storage [10], variations in the rancid-flavor compounds of milk under the general frozen-storage conditions have rarely been studied. Moreover, investigating the variations of rancidflavor compounds in human breastmilk under general frozen-storage conditions is important to not only better understand the possible impacts on the levels of flavor in breastmilk, but also to verify the appropriateness of the general recommendations for storing breastmilk.

Because human breastmilk is a dynamic and live fluid, numerous endogenous and exogenous factors affect the levels of milk lipolysis, e.g., gestational age, length of lactation, time of milking, nutrition states, hormonal treatment, and mastitis [12-14]. Therefore, this study adopted a repeated-measures design to determine the variations in rancid-flavor compounds of breastmilk under general frozen-storage conditions.

\section{Methods}

A repeated-measures study was conducted between April and September 2009 in Southern Taiwan. The study was approved by the institutional review board at a university hospital and informed consent was obtained before the research.

\section{Human breastmilk collection and storage}

Milk samples were obtained from ten healthy mothers of full-term infants with no mastitis, breast trauma, or abscesses, and no medication use one week prior to milk collection, which occurred between April 28 and May 26,2009 . The collection and storage procedures followed the general recommendations for handling human breastmilk for home use with health infants [2]. Milk was collected from one expression at the participating mothers' homes. After being expressed, the milk samples were stored in sterile polyethylene milk bags and immediately refrigerated. Within 30 minutes of collection, the milk was transported to the laboratory in an insulated cooler filled with ice packs, which kept these samples at $4^{\circ} \mathrm{C}-6^{\circ} \mathrm{C}$. Each participating woman provided nearly 150 $\mathrm{ml}$ in one pumping session. Because previous studies have indicated that longer periods of frozen storage causes more severe lipolysis in human breastmilk [15], each sample in this study was separated into three $50 \mathrm{ml}$ aliquots and stored in glass bottles: one as the fresh sample (refrigerated for less than 24 hours), the second as the 7-day frozen sample, and the third as the 30-day frozen sample. The fresh samples were immediately analyzed. To emulate the general practice of women freezing their breastmilk at home, the 7-day and 30-day samples were frozen and stored in the back area in the freezer of a typical household fridge (freezer temperature range of $-15^{\circ} \mathrm{C}$ to $-18^{\circ} \mathrm{C}$ ), which has separate doors for the refrigerator and freezer. After 7 and 30 days frozen storage, the frozen samples were completely thawed in the refrigerator for approximately 24 hours and then analyzed (Fig. 1).

\section{Human breastmilk analysis}

The rancid-flavor compounds, acid value (AV), overall FFAs, and volatile short and intermediate-chain FFAs [9, 16] were analyzed in the university's "Organic and Health food inspection laboratory", which has certification from the Ministry of Health and Welfare of Taiwan.

Acid value is a hydrolytic rancidity indicator of milk fat. AV is defined as the number of milligrams of potassium hydroxide $(\mathrm{KOH})$ required to neutralize the FFAs contained in one gram of fat [16]. To determine the AV of milk, the milk fat was firstly extracted via the Rose- 


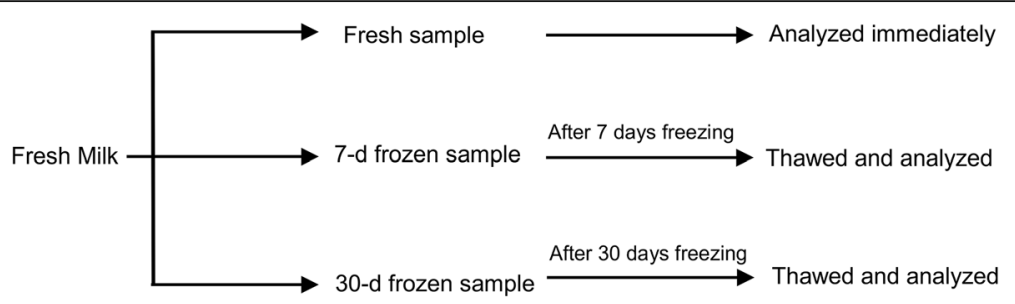

Fig. 1 The process of handling each breast milk sample

Gottlieb method [17]. After the milk fat was extracted, a mixture of ethyl ether and ethanol $(1: 1, \mathrm{v} / \mathrm{v})$ was used to dissolve it. Subsequently, two drop $1 \%$ phenolphthalein was added as colorimetric indicator and then the mixture was titrated with a $\mathrm{KOH}$ ethanol solution. When pink color was observed for at least 10 seconds, the volume of $\mathrm{KOH}$ used was recorded. The AV was calculated according to the formula: Acid value $=V_{K O H} \times M_{K O H} \times$ $56.1 / W_{\text {fat }}$, where $V_{K H O}$ is the volume of $\mathrm{KOH}$ used $(\mathrm{ml})$, $M_{K O H}$ is the molarity of the $\mathrm{KOH}$ solution, 56.1 is the molecular weight of $\mathrm{KOH}, W_{\text {fat }}$ is the milk fat in grams [16].

To analyze FFAs composition of milk, the processes of lipid extraction, lipid esterification, and gas chromatographic analysis were adapted. After milk fat was extracted via the Rose-Gottlieb method [17], two steps were then performed for the conversion of lipid-bound fatty acids and FFAs into fatty acid methyl esters (FAMEs) [18]. Heptane was used to dissolve the lipids after which sodium methoxide was added for transesterification of the glyceride-bound fatty acids into FAMEs. Hydrochloric acid then added to precipitate sodium glycerolate for the esterification of the FFAs into FAMEs. The resultant FAMEs were determined and quantified by gas chromatography (Shimadzu-17A, Tokyo, Japan) with a flame ionization detector and capillary column, which had an internal diameter of $0.53 \mathrm{~mm} \times 30 \mathrm{~mm}$ and a $1.0 \mathrm{~mm}$ film thickness.

\section{Statistical analysis}

To address the limited number of studies and the different milk treatments used therein, the FFAs changes between the fresh and 7-day frozen breastmilk among the first five milk samples collected in the current study were used for power analysis. G-power [19] was applied to estimate the required sample size. The sample size was calculated based on the effect size, which was calculated through the formula:

$$
\text { effect size }=\frac{\text { mean of FFAs7-day frozen }- \text { mean of FFAs } \text { fresh }_{\text {fres }}}{S D_{\text {fresh }}}
$$

Where mean of FFAs 7 - day frozen $=12.68$, mean of FFAs fresh $=5.87$, and SD fresh $=3.38$ ). Given an effect size of 2.01and an alpha level of 0.05 , a sample size of 10 for each group was necessary to achieve $80 \%$ power [20].

Data analyses included descriptive and inferential statistics. Descriptive statistics, including estimated mean, range, standard deviation for continuous variables, as well as percentages and frequencies for categorical variables, were tabulated and presented. With inferential statistics, considering the small sample size, a nonparametric Friedman test [21], which evaluates differences in medians among more than two groups, was adopted to compare the differences in the levels of $\mathrm{AV}$, overall FFAs and shortand intermediate-chain FFAs among the fresh, 7-day and 30-day frozen samples. In addition, Wilcoxon signed rank test [22] was then used as post-hoc test to determine which pairs of milk samples differ. Moreover, the characteristics of FFA based on different groups (fresh, 7-day frozen and 30-day frozen) were graphically displayed using box plots to visualize and compare the change of FFA values. All statistical tests were 2-sided and conducted with $\mathrm{R}$ version 3.3.1. [23] and statistical package for social sciences (SPSS) 17.0.

\section{Results}

The milk samples were donated from 10 healthy women with a mean age of 31 (range: 27-35 years old) and 124 days mean lactation. Most milk samples were expressed by pump, the fat content of which in the freshly expressed samples was $2.8 \pm 0.70 \%$ (Table 1 ).

In this study, the rancid-flavor compounds in breastmilk, AV, total FFAs, and two medium-chain FFAs, namely caproic acid (C10:0) and lauric acid (C12:0), were detected and all were found to increase with frozen-storage time. As shown in Fig. 2, the rate at which these compounds increased was seemingly faster between the fresh to 7 days frozen storage than that between the 7 to 30 days frozen storage.

Table 1 Characteristics of milk donors and samples $(N=10)$

\begin{tabular}{lll}
\hline Item & Range & Mean $\pm \mathrm{SD} / \%$ \\
\hline Age (years) & $27-35$ & $31 \pm 3.6$ \\
Length of lactation (days) & $34-180$ & $124 \pm 54$ \\
Milking by pump (n) & 8 & 80 \\
Fat content in milk (\%) & $2.02-4.34$ & $2.8 \pm 0.70$ \\
\hline
\end{tabular}




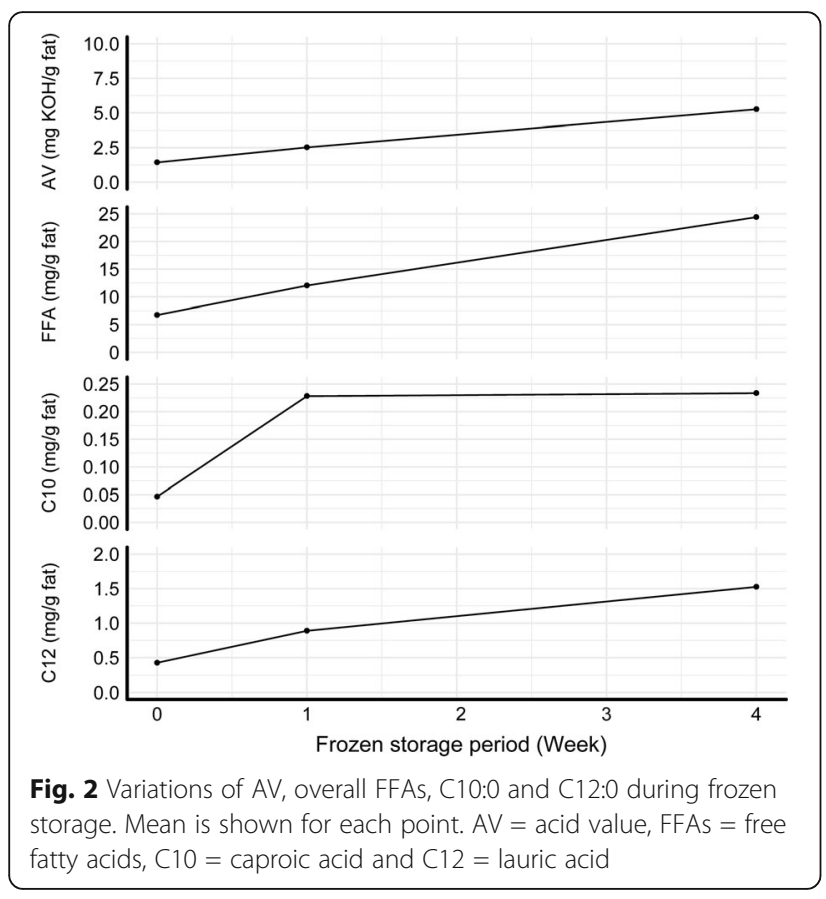

As presented in Fig. 3, the Friedman test revealed significant differences in overall FFAs amounts among the fresh, 7-day, and 30-day frozen samples, with the post-hoc test showing that FFAs concentrations in the 30-day frozen samples were significantly higher than

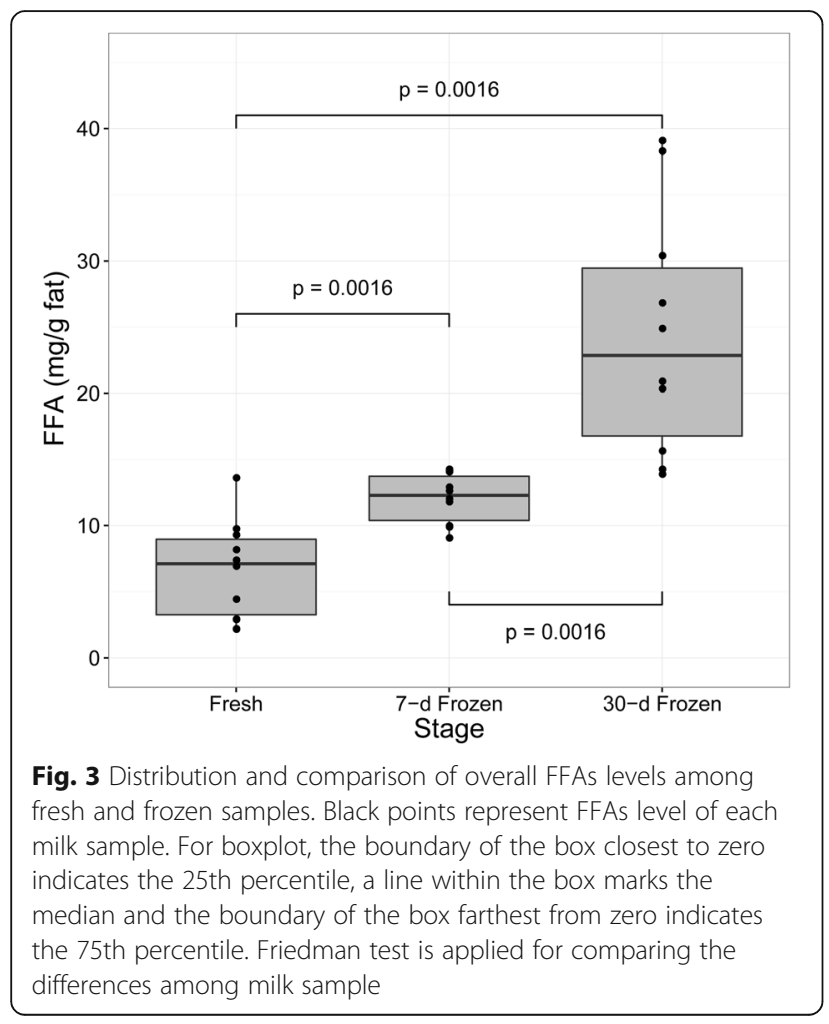

those in the fresh $(\mathrm{Z}=2.803, p<0.005)$ and 7-day frozen $(Z=2.803, p<0.005)$ samples.

Similarly, Table 2 depicts that the concentrations of AV, caproic acid (C10:0), and lauric acid (C12:0) revealed significant differences between the fresh and frozen samples. The post-hoc analysis indicated that $\mathrm{AV}$ and the lauric acid (C12:0) levels in the 30-day frozen samples were significantly higher than in both the fresh samples $(\mathrm{Z}=2.803, p=.005)$ and 7 -day frozen samples $(\mathrm{Z}=$ 2.293, $p=.022$ ). The caproic acid concentration in the 30-day frozen sample was significantly higher than in the fresh sample $(\mathrm{Z}=2.599, p=.009)$; however, no significant difference was found in the two other pairs (i.e. fresh vs. 7-day frozen, $\mathrm{Z}=1.478, p=.139$; 7 -day vs. 30 day frozen, $\mathrm{Z}=1.784, p=.074$ ).

\section{Discussion}

This study revealed that the AV, total FFAs, and caproic acid (C10:0) and lauric acid (C12:0) of human breastmilk increased with storage time, and that concentrations in the 30-day frozen milk samples were significantly higher than those in the 7-day frozen and fresh milk samples. These findings indicate that breastmilk's rancid-flavor progressively developed with frozenstorage time.

Nevertheless, few studies have considered the flavor of human breastmilk; in contrast, the flavor quality of cow's milk is strongly emphasized in the dairy industry since it affects customer acceptance and, in turn, the earnings of dairy producers [24]. Therefore, to characterize the potential influence of the levels of FFAs, caproic acid, and lauric acid on the flavor of breastmilk, a comparison with the recommended sensory threshold of rancid flavor in dairy products was made. Early studies in dairy science have reported the relative levels of FFAs, caproic acid (C10:0), and lauric acid (C12:0) in terms of the sensory threshold for detecting the rancid flavor of milk [9, $25,26]$.The detectable threshold usually means the minimum levels of a certain flavor compound perceived by at least $50 \%$ of the adult panelists [27]; accordingly, the FFAS levels in good-tasting milk should be lower than such thresholds [28].

Due to different analytical methods and panelist training, previous studies have reported considerable variations in the FFAs levels required for the detection threshold of the rancid flavor in milk, with a range from 1 to $3.62 \mathrm{mEq} / 100$ fat [9]. In response, the International Dairy Federation (1987) concluded that the FFAs levels for detecting the rancid-flavor threshold is normally between 1.5 to $2.0 \mathrm{mEq} / 100$ fat for most adult consumers, with levels exceeding approximately $1.5 \mathrm{mmol} / \mathrm{L}$ reaching the unacceptable threshold $[29,30]$. Specifically, the levels of caproic acid and lauric acid in milk 
Table 2. Acid value, total FFAs, and medium-chain FFAs in fresh and frozen breastmilk $(N=10)$

\begin{tabular}{|c|c|c|c|c|c|c|}
\hline \multirow[t]{2}{*}{ Composition } & \multirow{2}{*}{$\begin{array}{l}\text { Fresh (1) } \\
\text { Mean } \pm \text { SD }\end{array}$} & \multirow{2}{*}{$\begin{array}{l}\text { 7-day Frozen (2) } \\
\text { Mean } \pm S D\end{array}$} & 30-day Frozen (3) & \multirow[t]{2}{*}{$x^{2 a}$} & \multirow[t]{2}{*}{$p$ value } & \multirow[t]{2}{*}{ Post hoc } \\
\hline & & & Mean \pm SD & & & \\
\hline \multirow[t]{3}{*}{$\mathrm{AV}$ (mg KOH/g fat) } & \multirow[t]{3}{*}{$1.43 \pm 0.81$} & \multirow[t]{3}{*}{$2.52 \pm 0.44$} & \multirow[t]{3}{*}{$5.26 \pm 1.99$} & \multirow[t]{3}{*}{20.0} & \multirow[t]{3}{*}{$<.001$} & $(2)>(1)^{* *}$ \\
\hline & & & & & & (3) $>(1)^{* *}$ \\
\hline & & & & & & (3) $>(2)^{* *}$ \\
\hline \multirow[t]{3}{*}{ FFAs (mg/g fat) } & \multirow[t]{3}{*}{$6.74 \pm 3.64$} & \multirow[t]{3}{*}{$12.03 \pm 1.90$} & \multirow[t]{3}{*}{$24.41 \pm 9.25$} & \multirow[t]{3}{*}{20.0} & \multirow[t]{3}{*}{$<0.001$} & $(2)>(1)^{* *}$ \\
\hline & & & & & & (3) $>(1)^{* *}$ \\
\hline & & & & & & (3) $>(2)^{* *}$ \\
\hline \multicolumn{7}{|l|}{ Medium-chain } \\
\hline Caproic acid (C10:0) & $0.05 \pm 0.04$ & $0.23 \pm 0.49$ & $0.23 \pm 0.15$ & 10.4 & 0.006 & (3) $>(1)^{* *}$ \\
\hline \multirow[t]{3}{*}{ Lauric acid (C12:0) } & \multirow[t]{3}{*}{$0.43 \pm 0.24$} & \multirow[t]{3}{*}{$0.89 \pm 0.33$} & \multirow[t]{3}{*}{$1.52 \pm 0.65$} & \multirow[t]{3}{*}{16.2} & \multirow[t]{3}{*}{$<0.001$} & $(2)>(1)^{* *}$ \\
\hline & & & & & & (3) $>(1)^{* *}$ \\
\hline & & & & & & $(3)>(2)^{*}$ \\
\hline
\end{tabular}

AV Acid values, FFAs Free fatty acids

${ }^{\mathrm{a}}$ Result of Friedman test; ${ }^{\mathrm{b}}$ Result of Wilcoxon signed rank test

${ }^{*} p<0.05,{ }^{* *} p<0.01$

required for reaching the rancid-flavor threshold were reported to be 7 and 8 ppm, respectively [24].

To compare with the aforementioned flavor thresholds, the values of AV and FFAs in this study were converted into the same units. The AV of fresh, 7-day frozen, and 30-day frozen milk samples in this study were approximately 2.55, 4.49, and $9.19 \mathrm{mEq} / 100 \mathrm{~g}$ fat, respectively (unit conversion formula: $1 \mathrm{mg} \mathrm{KOH} / \mathrm{g}$ fat = $1 \times 100 \div 56.1 \mathrm{mEq} / 100 \mathrm{~g}$ fat; where $56.1=$ molecular weight of $\mathrm{KOH})$. In addition, the FFAs levels of the fresh, 7-day frozen, and 30-day frozen milk samples in this study were approximately $0.73,1.32$, and 2.63 $\mathrm{mmolL}^{-1}$, respectively (unit conversion formula: $1 \mathrm{mg} /$ $\mathrm{g}$ fat $=1 \times 28 / 260 \mathrm{mmolL}^{-1}$; where $28=\mathrm{g}$ fat contained in per liter human breastmilk of this study, $260=$ average molecular weight of FFAs estimated by the FFAs profile obtained in this study). These levels indicate that the flavor of fresh milk may already reach the rancidflavor detection threshold, and that the flavor of frozen milk may easily exceed the unacceptable rancid-flavor threshold. When focusing on the representative compounds of rancid flavor, we found that caproic acid concentrations in fresh and frozen breastmilk seemed to be below the detection levels (approximate values: fresh: 1.4 ppm, 7-day frozen: 6.44 and 30-day frozen: 6.44 ppm; unit conversion formula : $1 \mathrm{mg} / \mathrm{g}$ fat $=1 \times 28 \mathrm{ppm}$; where 28 = $\mathrm{g}$ fat contained in per liter human breastmilk of this study). In contrast, the lauric acid levels in the fresh and frozen breastmilk were much higher than the detection threshold for rancid flavor (approximate values: fresh: 12.04, 7-day frozen: 24.92, and 30-day frozen: $42.56 \mathrm{ppm})$.

Based on this comparison, this study found that the rancid flavor of the fresh breastmilk samples had already reached the rancid-flavor detection threshold for adults (2.55 mEq/100 fat); meanwhile, the FFAs levels in the 7-day (4.49 mEq/100 fat) samples far exceeded it and the 30-day frozen milk samples $(2.63 \pm 1.0$ $\mathrm{mmol} / \mathrm{L}$ ) reached intolerance level. In addition, lauric acid, which has unclean and soapy flavor attributes [25], may be the main contributor to the rancid flavor of human breastmilk.

Our study revealed that human breastmilk seems to be susceptible to lipolysis, even when fresh, which accords with findings of other studies. For example, Lavine \& Clark (1987) showed that the FFAs levels in fresh breastmilk were approximately $2.94 \mathrm{mEq} / 100 \mathrm{~g}$ fat $(0.23 \pm$ $0.087 \mathrm{mg} / \mathrm{ml}$ ) [15]. Slutzah [11] found that the FFAs concentrations in fresh breastmilk analyzed within 2.4 \pm 1.2 hours of expression were approximately $4.48 \mathrm{mEq} /$ $100 \mathrm{~g}$ fat $(0.35 \mathrm{mg} / \mathrm{ml})$. Another more recent study also indicated that human milk appears to be more susceptible to changes in flavor than bovine milk after frozen storage [8]. Therefore, the principle of FFA levels being lower than the detection threshold of the rancid flavor in milk may not be appropriate for direct application in assessing human breastmilk flavor. Moreover, one point worth noting was that the sensory threshold of detecting the rancid flavor in milk was based on adult panelists. However, previous studies have shown that infants' flavor sensitivity may differ from adults because even the protein hydrolysate formulas (PHF), which are perceived as extremely unpleasant for adults, are generally acceptable for infants younger than three months or those previously exposed to PHF [31]. This implies that age and learning experience affect infants' acceptance of food flavors [32]. Therefore, despite rancid-milk flavor being described as foul and objectionable by adults [27], it may 
be acceptable by infants, especially those younger than three months old.

Based on our findings, we assume that the FFAs levels found in fresh breastmilk not only facilitate fat digestion for infants but also provide learning experiences for infants so as to facilitate becoming accustomed to the rancid flavor in frozen breastmilk. However, despite the lack of evidence indicating that milk lipolysis alters nutritive components in human breastmilk [3], it was noted that extreme lipolysis in breastmilk may increase the probability of infants refusing it, as demonstrated in Hung's study [6]. Therefore, we recommend that when infants refuse thawed milk, mothers could try to provide freshly expressed breastmilk or breastmilk frozen less than 7 days. Concurrently, the traditional principle of breastmilk use, "use the oldest milk in the refrigerator or freezer first" might be best implemented only when it has been frozen for less than 7 days to avoid infant feeding stress. Moreover, we recommend future studies explore methods for decreasing the rate of breastmilk lipolysis to prolong its acceptable flavor. Although pasteurizing has been proposed to reduce milk lipolysis by inactivating the lipase $[9,11]$, the appropriate procedure for women to pasteurize their milk safely requires further study.

As a preliminary study exploring the rancid-flavor compounds variations of human breastmilk under general frozen-storage conditions, several limitations are acknowledged. The first is that to include more women to participate this study was not easy because providing breastmilk for study may affect their infants' feeding. Therefore, only 10 women with 30 milk samples were included in this study, which may affect the generalization of the findings. The second is that a direct sensory-perception test was not conducted in this study; accordingly, an assessment of infants' acceptable thresholds of the rancid flavor in frozen breastmilk was not performed. Future studies could combine observations of infants feeding behaviors with quantitative analysis of flavor compounds to determine infants' acceptable threshold for the rancid flavor of breastmilk. A third limitation is that there are many other reactions that can cause abnormal flavors in milk, e.g. lipid oxidation and proteolysis [27]; however, this study preliminarily focused on variations in the levels of breastmilk lipolysis during frozen storage. To extend this work, future studies could apply organoleptic testing through a trained panel and also analyze the objective compounds to explore the effects of lipid oxidation or proteolysis on breastmilk flavor under general storage conditions.

\section{Conclusions}

Human breastmilk lipolysis increases in tandem with frozen-storage duration, which intensifies the rancid flavor in breastmilk. Lipolysis of 7-day frozen breastmilk far exceeded the threshold for detecting rancid flavor in dairy products, while the lipolysis in the 30day frozen milk reached the intolerable level. To prevent infant feeding stress, we suggest that human breastmilk frozen and stored under general conditions be preferably consumed within 7 days. Moreover, the traditional recommendation of breastmilk use, namely "use the oldest milk in the refrigerator or freezer first", might be best implemented only when the breastmilk has been frozen for less than 7 days. Accordingly, since breastmilk flavor modulates infants' intake of breastmilk, future studies should explore methods for slowing breastmilk lipolysis to maintain its fresh flavor and extend its storability.

\section{Abbreviations \\ AV: Acid value; FFAs: Free fatty acids; KOH: Potassium hydroxide; FAMEs: Fatty acid methyl esters; PHF: Protein hydrolysate formulas}

\section{Acknowledgements}

We thank all participants in this study for their cooperation and for providing their precious breast milk.

\section{Funding}

This study was supported by the Cheng-Hsing Medical Foundation.

\section{Availability of data and materials \\ Detailed raw data may be available upon request from the corresponding author.}

\section{Authors' contributions}

$\mathrm{HYH}, \mathrm{YYH}$, and YJC conceived and designed the study; HYH and PFS contributed to the analysis and interpretation of the data; $\mathrm{HYH}$ wrote the draft of the manuscript; YYH, PFS, and YJC reviewed the manuscript critically for important intellectual content; YJC provided technical and material support and final approval of the version to be published. All authors read and approved the final manuscript.

\section{Ethics approval and consent to participate}

This study was conducted after approval by the Institutional Review Board of the National Cheng Kung University Hospital, Tainan, Taiwan. Informed written consent was obtained from the participating women.

\section{Consent for publication}

Not applicable.

\section{Competing interests}

The authors declare that they have no competing interests.

\section{Publisher's Note}

Springer Nature remains neutral with regard to jurisdictional claims in published maps and institutional affiliations.

\section{Author details}

${ }^{1}$ Department of Nursing \& Institute of Allied Health Sciences, College of Medicine, National Cheng Kung University, No. 1, Daxue Rd., East Dist., Tainan, Taiwan. 'Department of Statistics, National Cheng Kung University, Tainan, Taiwan. ${ }^{3}$ Nursing Department, National Cheng Kung University Hospital, Tainan, Taiwan. 
Received: 21 February 2017 Accepted: 20 February 2018

Published online: 02 March 2018

\section{References}

1. American Academy of Pediatrics. Breastfeeding and the use of human breast milk. Pediatrics. 2012;129:e827-41.

2. American Academy of Pediatrics and American College of Obstetricians and Gynecologists. Breastfeeding handbook for physicians. Elk Grove Village: American Academy of Pediatrics and American College of Obstetricians and Gynecologists; 2006.

3. Academy of Breastfeeding Medicine Protocol C, Eglash A. ABM clinica protocol \#8: human breastmilk storage information for home use for fullterm infants (original protocol March 2004; revision \#1 March 2010). Breastfeed Med. 2010;5:127-30.

4. Friend BA, Shahani KM, Long CA, Vaughn LA. The effect of processing and storage on key enzymes, B vitamins, and lipids of mature human breastmilk. I. Evaluation of fresh samples and effects of freezing and frozen storage. Pediatr Res. 1983;17:61-4.

5. Garcia-Lara NR, Escuder-Vieco D, Garcia-Algar O, De la Cruz J, Lora D, PallasAlonso C. Effect of freezing time on macronutrients and energy content of breastmilk. Breastfeed Med. 2012;7:295-301.

6. Hung HY, Hsu YY, Chang YJ. Comparison of physiological and behaviora responses to fresh and thawed breast milk in premature infant-a preliminary study. Breastfeed Med. 2013:8:92-8.

7. Jensen RG. Handbook of milk composition. San Diego: Academic Press; 1995

8. Spitzer J, Doucet S, Buettner A. The influence of storage conditions on flavour changes in human breastmilk. Food Qual Prefer. 2010;21:998-1007.

9. Deeth HC, Fitz-Gerald CH. Lipolytic enzymes and hydrolytic rancidity. In: Fox PF, McSweeney P, editors. Advanced dairy chemistry, vol. 2. New York: Springer; 2006. p. 481-529.

10. Berkow SE, Freed LM, Hamosh M, Bitman J, Wood DL, Happ B, Hamosh P. Lipases and lipids in human breastmilk: effect of freeze-thawing and storage. Pediatr Res. 1984;18:1257-62.

11. Slutzah M, Codipilly C, Potak D, Clark R, Schanler R. Refrigerator storage of expressed breast milk in the neonatal intensive care unit. J Pediatr. 2010; 156:26-8.

12. Ahrné $L, B j o ̈ r c k ~ L$. Lipolysis and the distribution of lipase activity in bovine milk in relation to stage of lactation and time of milking. J Dairy Res. 1985; 52:55-64.

13. Freed LM, Berkow SE, Hamosh P, York CM, Mehta NR, Hamosh M. Lipases in human breastmilk: effect of gestational age and length of lactation on enzyme activity. J Am Coll Nutr. 1989;8:143-50.

14. Ma Y, Ryan C, Barbano DM, Galton DM, Rudan MA, Boor KJ. Effects of somatic cell count on quality and shelf-life of pasteurized fluid milk. J Dairy Sci. 2000;83:264-74.

15. Lavine M, Clark RM. Changing patterns of free fatty acids in breast milk during storage. J Pediatr Gastroenterol Nutr. 1987;6:769-74.

16. Nollet LML, Toldra F. Handbook of dairy food analysis. Boca Raton: CRC Press; 2009

17. Association of Official Agricultural Chemists. AOAC official method 905.02 for fat in milk, Roese-Gottlieb method. In: Horwitz W, editor. Official Methods of Analysis of the Association of Official Agricultural Chemists. 17th ed. USA: AOAC International; 2000

18. De Jong C, Badings HT. Determination of free fatty acids in milk and cheese procedures for extraction, clean up, and capillary gas chromatographic analysis. J High Resolut Chromatogr. 1990;13:94-8.

19. Faul F, Erdfelder $E$, Lang AG, Buchner A. G* Power 3: a flexible statistical power analysis program for the social, behavioral, and biomedical sciences. Behav Res Methods. 2007:39:175-91.

20. Cohen J. Statistical power analysis for the behavioral sciences. London: Lawrence Erlbaum Associates; 1988.

21. Friedman $M$. The use of ranks to avoid the assumption of normality implicit in the analysis of variance. J Am Stat Assoc. 1937;32:675-701.

22. Wilcoxon F. Individual comparisons by ranking methods. Biometrics. 1945; 1:80-3.

23. $\mathrm{R}$ Core Team. R: a language and environment for statistical computing. $\mathrm{R}$ Foundation for Statistical Computing, Vienna, Austria. 2016. https:// www.r-project.org. Accessed 16 Feb 2016.

24. Cadwallader KR, Singh TK. Flavours and off-flavours in milk and dairy products. In: Fox PF, McSweeney P, editors. Advanced dairy chemistry, vol. 3. New York: Springer; 2009. p. 631-90.
25. Jeon IJ. Undesirable flavors in dairy products. In: Saxby MJ, editor. Food taints and off-flavours. New York: Blackie Academic and Professional; 1996. p. 139-67.

26. Santos MV, Ma Y, Caplan Z, Barbano DM. Sensory threshold of offflavors caused by proteolysis and lipolysis in milk. J Dairy Sci. 2003;86: 1601-7.

27. Clark S, Costello M, Drake M, Bodyfelt F. The sensory evaluation of dairy products. New York: Springer; 2009.

28. Wolf IV, Bergamini CV, Perotti MC, Hynes ER. Sensory and flavor characteristics of milk, production, composition and health. In: Park YW Haenlein G, editors. Milk and dairy products in human nutrition. Oxford: John Wiley and Sons; 2013. p. 310-29.

29. Deeth HC. Lipoprotein lipase and lipolysis in milk. Int Dairy J. 2006:16:555-62.

30. International Dairy Federation. Significance of lipolysis in the manufacture and storage of dairy products. Brussels: International Dairy Federation; 1987.

31. Mennella JA, Beauchamp GK. Understanding the origin of flavor preferences. Chem Senses. 2005;3(Suppl 1):i242-3.

32. Beauchamp GK, Mennella JA. Flavor perception in human infants: development and functional significance. Digestion. 2011;83(Suppl 1):1-6.

\section{Submit your next manuscript to BioMed Central and we will help you at every step:}

- We accept pre-submission inquiries

- Our selector tool helps you to find the most relevant journal

- We provide round the clock customer support

- Convenient online submission

- Thorough peer review

- Inclusion in PubMed and all major indexing services

- Maximum visibility for your research

Submit your manuscript at www.biomedcentral.com/submit 